\title{
Research on Knowledge Transfer Influencing Factors in Software Process Improvement
}

\author{
Jiangping Wan ${ }^{1,2}$, Qingjing Liu ${ }^{1}$, Dejie $\mathrm{Li}^{1}$, Hongbo $\mathrm{Xu}^{3,4}$ \\ ${ }^{1}$ School of Business Administration, South China University of Technology, Guangzhou, China; ${ }^{2}$ Institute of Emerging Industrializa- \\ tion Development, South China University of Technology, Guangzhou, China; ${ }^{3}$ School of Computer Science and Engineering, South \\ China University of Technology, Guangzhou, China; ${ }^{4}$ Guangzhou O-Engineer Information Technology Ltd, Guangzhou, China. \\ Email: scutwjp@126.com,ruthy07@163.com,ab23456@163.com
}

Received October $28^{\text {th }}, 2009$; revised November $17^{\text {th }}, 2009$; accepted November $25^{\text {th }}, 2009$.

\begin{abstract}
Knowledge transfer model of software process improvement (SPI) and the conceptual framework of influencing factors are established. The model includes five elements which are knowledge of transfer, sources of knowledge, recipients of knowledge, relationship of transfer parties, and the environment of transfer. The conceptual framework includes ten key factors which are ambiguity, systematism, transfer willingness, capacity of impartation, capacity of absorption, incentive mechanism, culture, technical support, trust and knowledge distance. The research hypotheses is put forward. Empirical study concludes that the trust relationship among SPI staffs has the greatest influence on knowledge transfer, and organizational incentive mechanism can produce positive effect to knowledge transfer of SPI. Finally, some suggestions are put forward to improve the knowledge transfer of SPI: establishing a rational incentive mechanism, executing some necessary training to transfer parties and using software benchmarking.
\end{abstract}

Keywords: Software Process Improvement, Knowledge Transfer, Influence Factors, Pattern

The software process is the set of tools, method, and practices we use to produce a software product. The objectives of software process improvement (SPI) are to process produce products according to plan while simultaneously improving the organization's capability to produce better products [1]. The six basic principles of SPI by Watts S. Humphrey are as follows: 1) Major changes to the software process must start at the top; 2) Ultimately, everyone must be involved; 3) Effective change requires a goal and knowledge of the current process; 4) Change is continuous; 5) Software process changes will not be retained without conscious effort and periodic reinforcement; 6) Software process improvement requires investment [1]. Alfonso Fuggetta argues that the scope of software improvement methods and models should be widened in order to consider all the different factors affecting software development activities. We should reuse the experiences gained in other business domains and in organizational behavior research. Statistics is not the only source of knowledge. We should

\footnotetext{
${ }^{*}$ This research was supported by Key Project of Guangdong Province Education Office (06JDXM63002), Soft Science project of Guangdong Province (2007B070900026), NSF of China (70471091), and QualiPSo (IST-FP6-IP-034763)
}

also appreciate the value of qualitative observations [2]. Wan Jiangping argues that managers should think deeply into their think processes. The following issues in software organization can be resolved with SPI: 1) The processes and their principles for how to inherit and acquire others' knowledge; 2) The processes and their principles for conversion knowledge into their capability [3]. Literature 6 describes a repository of 400 process improvement experiments and presents patterns that help organizations plan their improvement initiatives [4].

\section{Introduction}

\subsection{Organization Knowledge in Software Process Improvement}

Organizational knowledge creation is the process of making available and amplifying knowledge created by individuals as well as crystallizing and connecting it to an organization's knowledge system [5]. Software organization is a highly knowledge-intensive enterprise, knowledge transfer is critical for software enterprise. It is obvious that software process is also an organizational knowledge intensive learning process and needed to be supported with knowledge management [6]. 
Sandra A. Slaughter and Laurie J. Kirsch conceptualize knowledge transfer portfolios in terms of their composition (the types of mechanisms used) and their intensity (the frequency with which the mechanisms are utilized). They hypothesize the influence of organizational design decisions on the composition and intensity of knowledge transfer portfolios for SPI. They then posit how the composition and intensity of knowledge transfer portfolios affect performance improvement. Their findings indicate that a more intense portfolio of knowledge transfer mechanisms is used when the source and recipient are proximate, when they are in a hierarchical relationship, or when they work in different units [7]. Literature 8 includes: 1) A knowledge management framework for SPI; 2) An innovative knowledge modeling and control approaches; 3) Mining and retrieval approaches on the software process assets; 4) A knowledge management for SPI.

\subsection{Knowledge Transfer}

Bloodgood considers knowledge transfer as knowledge transfer and transmit among various organizations and individual [9]. Argote considers enterprise knowledge transfer as a process that one organization's experiences impact on other's organizational action. It is that knowledge change or change knowledge recipients' behavior [10]. Davenport considers knowledge transfer as unified process which consists of both knowledge transfer process and knowledge absorbs process [11]. The effective knowledge transfer is that transfer knowledge is reserved [12]. Ingram considers knowledge transfer as process sharing knowledge in organization through various channels in order to make use of extant knowledge effectively [13]. Dong-Gil Ko et al. consider knowledge transfer as transmitting process in which knowledge transfer from owners to recipients for their learning and application [14].

In our understanding, knowledge transfer includes three aspects which are the process spreading from owners to recipients, activities occurring under contextualization and special goal. But the ultimate goal is to make the knowledge of the owners be the recipients' and narrow the knowledge gap between owners and recipients so as to promote the co-development of individuals and organizations. We define knowledge transfer as the process making knowledge transferring from the source of knowledge to recipients in contextualization.

\subsection{Knowledge Transfer Model}

The knowledge transfer model mainly includes process model and factors model. The process model is a model dividing knowledge transfer into different stages. The representative process models are Nonaka knowledge spiral model [15], Szulanski four stages model [12], and Gilbert\&Cordey-Hayes five steps model. While factors model bases on factors in the process the knowledge transfer [16]. The representative ones of it are the four factors model invented by Jeffrey L. Cummings and Bing-Sheng Teng which includes sources of the knowledge, recipients of the knowledge, knowledge and context and transfer framework invented by Vito Albino et $a l$. including transfer subject, context, content, and transfer media. Jeffrey L. Cummings and Bing-Sheng Teng's factors model is applied in this study [17].

\subsection{Knowledge Transfer Influencing Factors}

Knowledge transfer influencing factors are in the following [18]: 1) Characteristics of knowledge transferred include causal ambiguity and unprovability. 2) Characteristics of source of knowledge include knowledge providers shortage of motivation to transfer knowledge and unbelieving. The knowledge owners will not sharing knowledge with others because they are afraid losing knowledge possession, superiority complex, right and status and so on, lack time to sharing knowledge with others and couldn't proper reward and return on knowledge sharing. When the experts are not discovered and believed, their suggestions may be rejected and more challenged. 3) Characteristics of recipient of knowledge include knowledge recipients' both absorbing capability and keeping capability. It is very important for the knowledge recipient's capability to absorb others' knowledge and integrate into individual knowledge on the condition he will accept the knowledge developed by other. 4) Characteristics of context include barren organizational context and arduous relationship. Both will impart on knowledge transfer.

\section{Research Model and Hypotheses}

The knowledge transfer model of SPI, which includes five factors involving knowledge transferred, source of knowledge, recipient of knowledge, relationship of two parties and context. Besides is proposed, we consider knowledge transfer of SPI as a process which includes transmission, absorption and feedback (Figure 1). Knowledge transferred between source of knowledge and recipient of knowledge in the SPI must experience three stages. The stage of transmission is to transmit knowledge from source of knowledge to recipients of knowledge. The stage of absorption is about processing, sorting, and absorption process with their own mental model when recipients receive new knowledge. In stage of feedback, recipients of knowledge constantly communicate and feedback with source of knowledge in the process of absorption to master the knowledge transferred by the owners of knowledge. Thus, based on knowledge transfer model of SPI, we propose conceptual framework of ten key influencing factors of knowledge transfer in SPI. The influencing factors include ambiguity, systematism, transfer willingness, capacity of impartation, capacity of absorption, incentive mechanism, culture, technical 


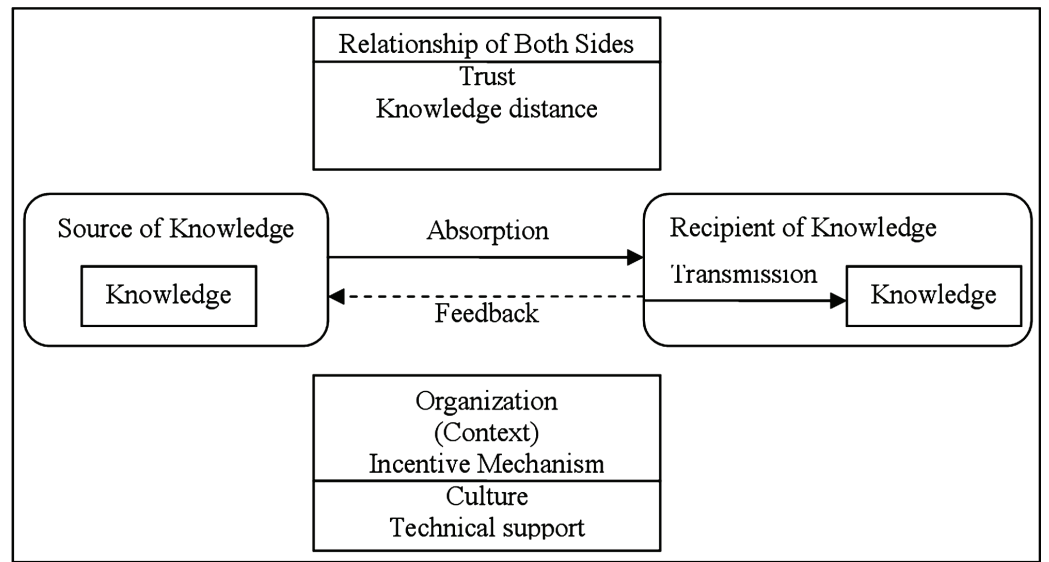

Figure 1. Knowledge transfer model of SPI

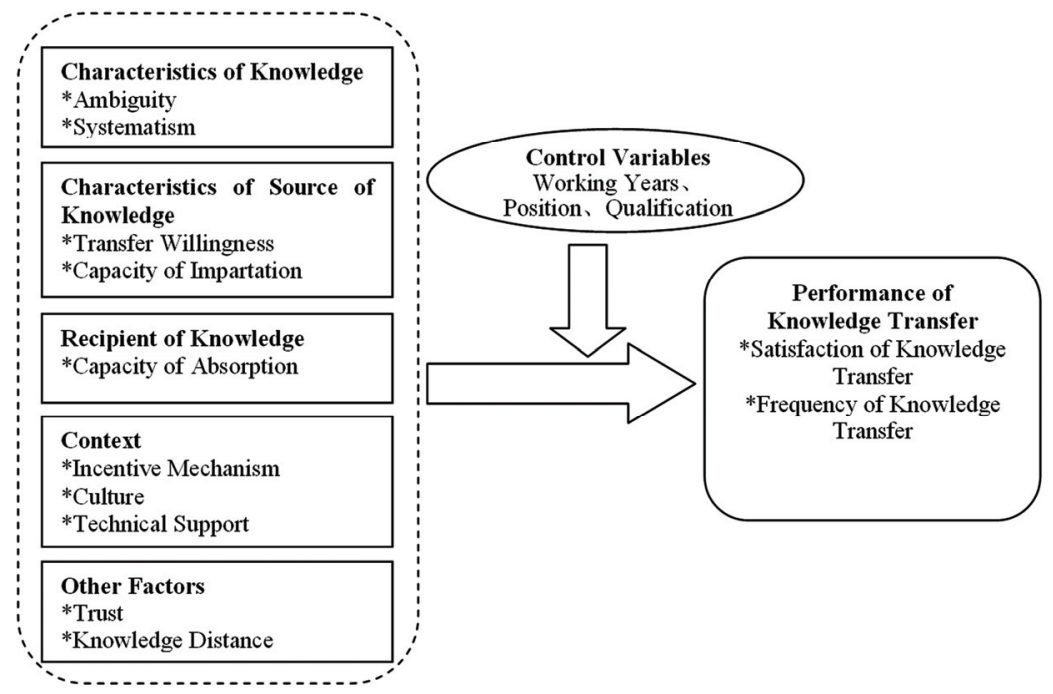

Figure 2. Conceptual framework of influencing factors

support, trust and knowledge distance (Figure 2). Then we propose our research hypotheses based on knowledge transfer model and conceptual framework of knowledge transfer influencing factors (Table 1).

\section{Research Design}

\subsection{Scale Design}

We studied the variable indicators on a five-point Likert-type scale. The questionnaire of pre-investigation was divided into four parts. The first part was questionnaire direction, including questionnaire background and introduction of basic information. The second part was the basic information of respondents, including gender, age and qualifications of respondents; the third part was the main body of the questionnaire, mainly about the issues of influence factors, including a total of 46 items. The last part with regard to performance of knowledge transfer involved a total of 4 items. After the completion of the pre-prepared questionnaire, we pre-prepared questionnaire in a small number of target groups by e-mail.
We issued 10 questionnaires and six were received. In the end, we received a total of 114 questionnaires, of which eight were invalid, 106 were valid.

\subsection{Data Collection}

All the samples of our research are SPI staffs from Guangdong Software Organization who mainly concentrate in Guangzhou, Shenzhen and Zhuhai. From the job level of respondents, senior manager accounts for $8.5 \%$, the percent of project manager is $8.5 \%$, while the general staff contributes the large percent of $66.0 \%$. From the qualifications of respondents, undergraduate accounts for about $74.5 \%$, graduate $25.5 \%$. From the age of respondents, age ranged from 24 to 30 accounts for the largest proportion of $67.9 \%$, age bellow 24 and above 30 are $14.2 \%$ and $17.9 \%$ respectively. From the staff size of process improvement of investigated company, staff size bellow 10 comes up to $63.2 \%$, staff size between 11 to 30 accounts for $17.9 \%$, size above 31 reaches $18.9 \%$. It can clearly be seen that, the samples have representative to some extent, and are suitable for the next phase of data analysis. 
Table 1. Research hypotheses

\begin{tabular}{|c|c|}
\hline Items & Hypotheses \\
\hline & H1: Ambiguity of knowledge transferred has correlation with performance of knowledge transfer \\
\hline Ambiguity & H1a: Ambiguity of knowledge transferred has negative correlation with satisfaction of knowledge transfer \\
\hline & H1b: Ambiguity of knowledge transferred has negative correlation with frequency of knowledge transfer \\
\hline & H2: Systematism of knowledge transferred has correlation with performance of knowledge transfer \\
\hline Systematicness & H2a: Systematism of knowledge transferred has negative correlation with satisfaction of knowledge transfer \\
\hline & H2b: Systematism of knowledge transferred has negative correlation with frequency of knowledge transfer \\
\hline & H3: Transfer Willingness of source of knowledge has correlation with performance of knowledge transfer \\
\hline $\begin{array}{l}\text { Transfer } \\
\text { ingness }\end{array}$ & $\begin{array}{l}\text { H3a: Transfer Willingness of source of knowledge has positive correlation with satisfaction of knowledge transfer } \\
\text { H3b: Transfer Willingness of source of knowledge has positive correlation with frequency of knowledge transfer } \\
\text { H4: Capacity of Impartation of source of knowledge has correlation with performance of knowledge transfer }\end{array}$ \\
\hline $\begin{array}{l}\text { Capacity of Im- } \\
\text { partation }\end{array}$ & $\begin{array}{l}\text { H4a: Capacity of Impartation of source of knowledge has positive correlation with satisfaction of knowledge transfer } \\
\text { H4b: Capacity of Impartation of source of knowledge has positive correlation with frequency of knowledge transfer } \\
\text { H5: Capacity of Absorption of recipient of knowledge has correlation with performance of knowledge transfer }\end{array}$ \\
\hline $\begin{array}{l}\text { Capacity of Ab- } \\
\text { sorption }\end{array}$ & $\begin{array}{l}\text { H5a: Capacity of Absorption of recipient of knowledge has positive correlation with satisfaction of knowledge transfer } \\
\text { H5b: Capacity of Absorption of recipient of knowledge has positive correlation with frequency of knowledge transfer } \\
\text { H6: Incentive Mechanism of knowledge has correlation with performance of knowledge transfer }\end{array}$ \\
\hline Incentive & H6a: Incentive Mechanism has positive correlation with satisfaction of knowledge transfer \\
\hline Mechanism & $\begin{array}{l}\text { H6b: Incentive Mechanism has positive correlation with frequency of knowledge transfer } \\
\text { H7: Organizational culture has correlation with performance of knowledge transfer }\end{array}$ \\
\hline Culture & $\begin{array}{l}\text { H7a: Organizational culture has positive correlation with satisfaction of knowledge transfer } \\
\text { H7b: Organizational culture has positive correlation with frequency of knowledge transfer } \\
\text { H8: Technical Support of knowledge transfer has correlation with performance of knowledge transfer }\end{array}$ \\
\hline $\begin{array}{l}\text { Technical } \\
\text { port }\end{array}$ & $\begin{array}{l}\text { H8a: Technical Support of knowledge transfer has positive correlation with satisfaction of knowledge transfer } \\
\text { H8a: Technical Support of knowledge transfer has positive correlation with frequency of knowledge transfer } \\
\text { H9: Trust relationship between transmitter and recipient of knowledge has correlation with performance of knowledge transfer }\end{array}$ \\
\hline Trust & $\begin{array}{l}\text { H9a: Trust relationship between transmitter and recipient of knowledge has positive correlation with satisfaction of } \\
\text { knowledge transfer }\end{array}$ \\
\hline & $\begin{array}{l}\text { H9b: Trust relationship between transmitter and recipient of knowledge has positive correlation with frequency of knowledge transfer } \\
\text { H10: Knowledge Distance between transmitter and recipient of knowledge has curve correlation with performance of knowledge transfer }\end{array}$ \\
\hline $\begin{array}{l}\text { Knowledge Dis- } \\
\text { tance }\end{array}$ & $\begin{array}{l}\text { H10a: Knowledge Distance between transmitter and recipient of knowledge has curve correlation with staff's satisfac- } \\
\text { tion of knowledge transfer When knowledge distance is short, the correlation appears negative; as it becomes moderate, the } \\
\text { correlation appears positive and when knowledge distance is considerable long, and the correlation becomes negative again. } \\
\text { H10b: Knowledge Distance between transmitter and recipient of knowledge has curve correlation with staff's fre- } \\
\text { quency of knowledge transfer. When knowledge distance is short, the correlation appears negative; as it becomes moderate, } \\
\text { the correlation appears positive and when knowledge distance is considerable long, the correlation becomes negative again. }\end{array}$ \\
\hline Control Variables & H11: The individual's working years, position and qualification have influence on performance of knowledge transfer \\
\hline
\end{tabular}

\subsection{Result Analysis and Explanation}

\subsubsection{Statistical Result Analysis}

In order to ensure the scientific nature of the proposition certification, it is necessary to test the reliability and validity of the measure model. First, all variables' Cronbach's coefficient are significantly higher than the minimum threshold 0.70 , factor analysis and confirmatory factor analysis are all met reference standards, so we can judge it has internal validity. Second, for all indicators, standardized loading factors are also higher than that of the recommended minimum critical level 0.50 , the statistical value of Battelle is a much smaller than 0.01. All of these indicate that all scales have highly convergent validity. The above shows that the research ha good external validity. Integrated the test of reliability and validity, the scales are reliable and effective which can be used to verify model assumptions.

Correlation analysis was executed between knowledge transfer performance and its influence factors by using SPSS16.0 statistical software, and the result was presented in Table 2. The result concludes that culture has no significant correlation to performance of knowledge transfer. Capacity of impartation and incentive mechanism has significantly positive correlation to performance of knowledge transfer at the significant level of 0.05 . While ambiguity has significantly negative correlation to performance of knowledge transfer at the significant level of 0.01 , the rest factors have significantly positive correlation to performance of knowledge transfer at the significant level of 0.01 . To avoid multiple co-linear problems, we defined values of knowledge 
transfer influence factors as independent variables, and executed regression analysis by step regression method when considering the causal relationship among knowledge transfer performance and its influence factors. The results are in Table 3.

From the overall regression effect with $\mathrm{F}=18.967, \mathrm{P}=$ 0.000 , regression equation has achieved a very significant level which indicates better regression effect. Meanwhile, variance expansion factor VIF had small value and multiple co-linear problems were not obvious. The adjusted determination coefficient is 0.339 indicating that three indicators of knowledge transfer influence can explain $33.9 \%$ of the total variance. The coefficient values of constant and variables are all less than 0.05 suggesting that they have significant meaning.

Table 2. Correlation analysis between influencing factors and performance of knowledge transfer

\begin{tabular}{lll}
\hline \multicolumn{1}{c}{ Performance } & $\begin{array}{l}\text { Performance of } \\
\text { edge Transfer }\end{array}$ & Knowl- \\
Influencing Factors & Correlation & Sig. \\
\hline L1 Ambiguity & $-.143^{*}$ & .000 \\
L2 Systematism & $-.109^{* *}$ & .000 \\
L3 Transfer Willingness & $.375^{* *}$ & .000 \\
L4 Capacity of Impartation & $.285^{*}$ & .0016 \\
L5 Capacity of Absorption & $.392^{* *}$ & .000 \\
L6 Incentive Mechanism & $.387^{* *}$ & .009 \\
L7 Culture & 213 & .159 \\
L8 Trust & $.558^{* *}$ & .000 \\
\hline
\end{tabular}

Table 3. Overall effect parameter by step regression

\begin{tabular}{lllllll}
\hline Model & $\mathrm{R}$ & $\mathrm{R}^{2}$ & $\begin{array}{c}\text { Adjusted } \\
\mathrm{R}^{2}\end{array}$ & $\begin{array}{c}\text { Stan- } \\
\text { dard } \\
\text { Error }\end{array}$ & $\mathrm{F}$ & Sig. \\
\hline 1 & .512 & .262 & .255 & .625 & 37.004 & .000 \\
2 & .560 & .313 & .300 & .606 & 23.495 & .000 \\
3 & .598 & .358 & .339 & .589 & 18.967 & .000 \\
\hline
\end{tabular}

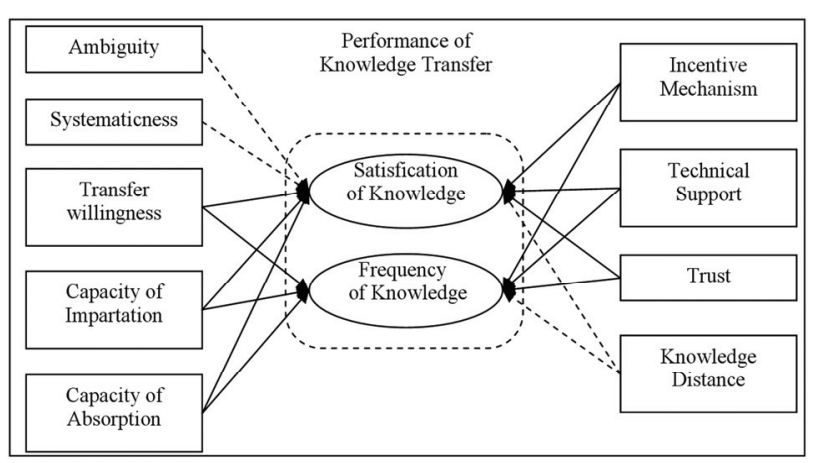

Figure 3. Influencing relationship of performance of knowledge transfer in software process
In order to clear the influence factors and their direction of the performance of knowledge transfer in SPI, we employed Figure 3 to express test results of Table 4 where the line indicated positive relationship and the dotted line negative relationship. From Table 4 and Figure 3 , we can conclude that the majority results of empirical research are consistent with our hypotheses and they are listed as follows: In the characteristics of knowledge, the ambiguity impacting on the performance of knowledge transfer mainly manifested on the satisfaction of transfer. The more ambiguous the source of knowledge is, the more time and energy will be spending when we express the knowledge out from source of knowledge. The systematization of knowledge impacting on the performance of knowledge transfer mainly indicates that it has a negative effect on the performance of knowledge transfer. The higher the systematism of knowledge is, the more difficult for transmitter of knowledge to express real meaning of the knowledge.

\subsubsection{Empirical Result Analysis}

According to the above statistical results, the test results of our hypotheses were concluded in Table 4.

In the characteristics of source knowledge, the transfer willingness of knowledge has significantly positive correlation to the satisfaction and frequency of knowledge transfer. In the characteristics of recipients of knowledge, the recipients' capacity of absorption has significantly positive influence on the effect of knowledge transfer.

In the environmental factors of knowledge transfer in SPI, the incentive mechanism has significantly positive influence on the satisfaction and frequency of knowledge transfer. The employees of the organization will retain their knowledge because they are worried about losing their authority when imparting their knowledge to others, if the organization does not take certain incentives.

In the relationship between source of knowledge and recipient of knowledge, trust relationship has the largest impact on the performance of knowledge transfer of all influence factors. This shows that trust relationship between source of knowledge and recipient of knowledge is the most basic factor of knowledge transfer in SPI. The curve relationship between knowledge distance and the performance of knowledge transfer is not obvious.

\section{Management Enlightment and Suggestion}

In order to improve the staffs' performance of SPI in practice, the following three aspects are necessary.

1) Establishing a reasonable incentive mechanism. Software organizations should build a reasonable incentive mechanism to enhance the transfer willingness of source of knowledge and consciousness of recipients of knowledge, it can also promote trust relationship between the source of knowledge and recipient of knowledge. To enhance the transfer willingness of source of knowledge, it is necessary to give corresponding material 
Table 4. Test results of hypotheses $(a=10, b=11)$

\begin{tabular}{llll}
\hline Hypothesis & Results & Hypothesis & Results \\
H1 & Support & H6a & Support \\
H1a & Support & H6b & Support \\
H1b & Not & H7 & Not Support \\
H2 & Support & H7a & Not Support \\
H2a & Support & H7b & Not Support \\
H2b & Not & H8 & Support \\
H3 & Support & H8a & Support \\
H3a & Support & H8b & Support \\
H3b & Support & H9 & Support \\
H4 & Support & H9a & Support \\
H4a & Support & H9b & Support \\
H4b & Not & HA & Not Support \\
H5 & Support & HAa & Not Support \\
H5a & Support & HAb & Not Support \\
H5b & Support & HB & Not Support \\
H6 & Support & & \\
\hline
\end{tabular}

or mental compensation and think highly of achievement of source of knowledge. Similarly, recipient of knowledge also need some encouragement to accept and use new knowledge. It can make the two sides of knowledge transfer participate actively by sharing their interests and therefore promote organizational SPI.

2) Carrying out necessary training for the both sides of knowledge transfer. After solving the transfer willingness of the two sides, the transfer capacity of source of knowledge, capacity of absorption of recipient of knowledge and distance between source of knowledge and recipient of knowledge have greater influence on knowledge transfer. Thus, software organizations need to give corresponding training for both of the two parties. They can employ external experts to train their staffs so that the staffs' capacity of imparting knowledge can gain improvement.

3) Using software benchmarking [19]. In pursuit of a capability model rating, software benchmarking (in our understanding, the benchmarking is standard best knowledge patterns, such as CMMI and SWEBOK [20], etc.) would help its process improvement and assessment effort. This benchmarking questionnaire can be grouped into five categories: a) Philosophy of implementation-how each company achieved CMMI compliance in terms of schedule, teams, and planning. b) Management commitment - the strength of institutional support for the process improvement effort. c) Cultural change and institutionalization-issues that arose regarding acceptance of the new process philosophy. d) Definition of organization-because the CMMI assessment is for specific organizations, these questions assessed the scope of their effort (for example, a section, company, or corporation). e) Objective evidence-the CMMI assessment process requires objective evidence that the new process is being followed, so these questions probed how each company collected evidence.

\section{Conclusions}

In this study, the knowledge transfer model of SPI and the conceptual framework of 10 key influence factors are established. Then research hypotheses are put forward. Empirical study concludes that the trust relationship among SPI staffs has the greatest influence on knowledge transfer, and organizational incentive mechanism can produce positive effect to knowledge transfer of SPI. We believed that the research is helpful for SPI practitioners to improve their performance of knowledge transfer.

\section{Acknowledgements}

Thanks for helpful discussion with Mr. Hou Yawen, Mr. Zhou qiyang, Mr. Li Jiangzhang, Mr. Nihao, Mr. Zhou Zhijun, and the hard work of my student Zeng Yonghua and Zheng Chuwei.

\section{REFERENCES}

[1] W. S. Humphrey, "Managing the software process," Reading, Addison-Wesley, MA, 1989.

[2] Alfonso Fuggetta, "Software process: A roadmap," Proceedings of the Conference on The Future of Software Engineering, Limerick, Ireland, pp. 25-34, June 04-11, 2000.

[3] J. P. Wan and J. M. Yang, "Knowledge management in SPI," Application Research of Computer, Vol. 19, No. 5, pp. 1-3, 2002.

[4] M. Blanco, P. Gutiérrez, and G. Satriani, "SPI patterns: Learning from experience," IEEE Software, Vol. 18, No. 3, pp. 28-35, 2001.

[5] I. Nonaka and G.g von Krogh, "Perspective-tacit knowledge and knowledge conversion: Controversy and advancement in organizational knowledge creation theory," Organization Science, Vol. 20, No. 3, pp. 635-652, 2009.

[6] J. P. Wan, "Research on software product support structure," Journal of Software Engineering and Applications, Vol. 2, No. 3, pp. 174-194, 2009.

[7] S. A. Slaughter and L. J. Kirsch, "The effectiveness of knowledge transfer portfolios in SPI: A field study," Information Systems Research, September 2006.

[8] X. G. Zhang, "Research on knowledge management technology in SPI," Chinese Academy of Sciences Doctoral Thesis, 2004.

[9] J. M. Bloodgood and W. D. Salisbury, "Understanding the influence of organizational change strategies on information technology and knowledge management strategies," Decision Support Systems, Vol. 31, No. 1, pp. 55-69, 2001.

[10] L. Argote, "Organizational learning: Creating, retaining and transfering knowledge," Kluwer Academic Publishers, pp. 143-189, 1999.

[11] T. H. Davenport and L. Prusak, "Working knowledge: 
How organization manage what they know," Harvard Business School Press, Boston, 1998.

[12] G. Szulanzki, "Exploring internal stickiness: Impediments to the transfer of best practice with the firm," Strategic Management Journal, Vol. 17, pp. 27-43, 1996.

[13] L. Argote and P. Ingram, "Knowledge transfer: A basis for competitive advantage in firms," Organizational Behavior and Human Decision Processes, Vol. 82, No. 1, pp. 150-169, 2000.

[14] D.-G. Ko, L. J. Kirsch, and W. R. King, "Antecedents of knowledge transfer from consultants to clients in enterprise system implementations," Management Information System Quarterly (Special Issue), Vol. 29, No. 1, pp. 5985, 2005.

[15] I. Nonaka and H. Takeuchi, "The knowledge creating company," Oxford University Press, New York, 1995.

[16] M. Gilbert and M. Cordey-Hayes, "Understanding the process of knowledge transfer to achieve successful technological innovation," Technovation, Vol. 16, pp. 301-312, 1996

[17] J. L. Cummings and B. S. Teng, “Transferring R \& D knowledge: The key factors affecting knowledge transfer success," Journal of Engineering and Technology Management, Vol. 20, pp. 39-68, 2003.

[18] M. Polanyi, "The study of man," Routledge \& Kegan, London, Vol. 12, 1957.

[19] G. C. Thomas and H. R. Smith, "Using structured benchmarking to fast-track CMM process improvement," IEEE Software, Vol. 18, No. 5, pp. 48-52, 2001

[20] P. Bourque, R. Dupuis, A. Abran, J.W. Moore, and L. Tripp, "The guide to the software engineering body of knowledge," IEEE Software, Vol. 16, No. 6, pp. 35-44, 1999. 